\title{
Acute Intraoperative Sustained Expansion (AISE) With or Without Fat Grafting Versus Local Flaps or Skin Grafts in Managing Facial Disfigurements
}

\author{
MOHAMED A. WAHSH, M.D. and AYMAN FIKRY, M.D. \\ The Department of General Surgery, Plastic \& Reconstructive Surgery Unit, Faculty of Medicine, Zagazig University
}

\begin{abstract}
Introduction: Facial disfigurement or cutaneous defect reconstruction might not be feasible without the aid of either skin grafts or local flaps, but they might have poor color and character match. Although chronic tissue expansion has succeeded to achieve an additional new skin with good match but it is not suitable in many cases. Acute Intraoperative Sustained Expansion (AISE) (rapid expansion) has been introduced as an alternate technique to aid in wound closure following the same strategy of preservation of a good tissue matching but in a single procedure, also when it is combined with fat grafting in case of contour irregularities, it enhance the aesthetic outcome.
\end{abstract}

Patients and Methods: This study included 27 patients with different facial disfigurements or facial cutaneous defects [post tumor excision, post-burn contracture release] located on nose, cheek, temple, or forehead, divided in two groups: Group A (Expansion group) included 13 patients those were treated with (AISE) by using total 21 intraoperative expansion by Foley catheters, five of these patients were managed with additional fat injection when associated with contour irregularities. In the other group, B (Flap/Graft group), 14 patients were managed by local flaps (11 patients) and skin grafts (3 patients).

Results: AISE showed success to gain additional skin surface area with each expansion site by dissection, expansion, or both with means of $14.2 \%, 16.5 \%$ and $30.7 \%$ of the involved basic relaxing skin surface area respectively with more aesthetic reliability if associated with fat grafting in cases with contour defects. However, expansion sites showed blood collection in $9,6 \%$, closure under tension in $9,6 \%$, partial wound dehiscence in $4.8 \%$. Also, the incidence of patients' dissatisfaction with the results was $23 \%$ of cases. Also, the group of local flaps and grafts cases showed poor color and texture matching to the surrounding area $25 \%$ in local flap cases and $100 \%$ in cases reconstructed by skin grafts, additional facial scars $100 \%$ in cases of local flaps, donor site complications and unsatisfactory imaging in $80 \%$ of cases, unnatural facial distortion $65 \%$, need for second procedure $28 \%$, wound dehiscence $22 \%$, partial graft loss $33 \%$ and flap viability problems $12 \%$.

Conclusion: Acute intraoperative sustained expansion could be considered as a reliable reconstructive tool in facial disfigurement, although it doesn't add skin as much as chronic expansion, but it is helpful in assisting closure of wounds without tension, that might otherwise be problematic, it suits small to moderate surface area cutaneous defects, it generates a good matched skin in single procedure, and gives more aesthetic outcome if combined with autologous fat grafting in cases with contour irregularities.

Key Words: Facial disfigurement - Acute tissue expansion - Fat grafting.

\section{INTRODUCTION}

Face is the keystone in perception of selfidentity which has a direct involvement in social and emotional communication, and poses a great importance both from functional and aesthetic point of view in a human life [1,2]. Patients suffering from facial disfigurements usually seek the plastic surgery clinic to restore function, comfort and appearance. The magnitude of the problem my varied from minimal dispersant scar to a debilitation condition. By the time, the plastic surgeon needs a wide verity of reconstructive tools, plenty of techniques and multiple surgical procedures. Even though, neither of these can be used as a single fully satisfactory solution [3].

The main potential issue in managing these cases is the lack of the skin with the same characteristics, anatomical and functional features [4]. For example, limited nearby donor skin in reconstruction of a post-burn disfigured scar may obligate the plastic surgeon to seek a suboptimal donor tissue as a full-thickness skin graft, local, or distant flaps which are common practices in managing these cases [5], as a result, incomplete color, nature and tissue texture matching between the donor and recipient site results in an obvious difference and less satisfactory results in a wide variety of patients [6]. 
Chronic tissue expansion had become a common practice in plastic surgery to compensate this tissue deficit with sound match [7]. This permits the surgeon to achieve generation of local tissue; to cover the cutaneous defect with a skin has the same color, texture and characters where the face needs this match [8]. At the same time, donor site morbidity can be minimized, sensate and highly specialized areas having hair or adnexal structures can be generated by the expansion process to be used $[\mathbf{9 , 1 0}]$.

Another type of expansion which was initially pioneered by Sasaki in 1987, it was repeated cycles of an acute intermittent expansion, it could stretch the skin beyond its natural limit, lessened the tension of wound closure and achieved an immediate reconstruction [11].

In this work, we aimed to evaluate AISE as an uncommon reconstructive tool in management of facial disfigurements, which are requiring skin add with or without subcutaneous volume add versus the commonly used local flaps and grafts.

The other target of this work was to delineate the concepts and the mechanism of action, and to differentiate between skin dissection and skin stretch and expansion adding role, and if providing a suitable benefit for contour add if associated with fat grafting $g$ as an additional touch up in managing associated contour defects.

\section{PATIENTS AND METHODS}

The present study was interventional study (pre-post), included twenty-seven $(n=27)$ patients, (11 males and 16 females) between July 2014 and May 2017, Patients were suffering from facial disfigurements; post-burn scar, post traumatic scar, post-inflammatory residual scar, residual deformity after infantile hemangioma and post-surgical tumor excision defects measuring ( 2 to $4 \mathrm{~cm}$ ) in diameter and located on nose, cheek, temple, or forehead skin, they were divided in in two groups.

Group A: (Expansion group) included 13 patients (age: 17-55 years, mean 34.4), they were treated with (AISE) by using totally 21 Foley catheters. One to three sites of expansion were applied in each case according to the tissue need and the suitability of surrounding areas. Five patients of this group were managed with additional multi-planar subcutaneous fat injection when associated with contour defects.
Group B: (Flap/Graft group): Included 14 patients (age 19-56 years, mean 36.9), they were managed by local random skin flaps ( 7 patients), and local pedicled skin flaps (5 patients), and skin grafts (3 patients).

After discussion of the procedures with all patients, and obtaining detailed informed consents from them, Pre-emptive antibiotics and general anaesthesia were routinely applied. Regarding the (Expansion group) patients, the operative and mathematical parameters of AISE we have measured and evaluated.

\section{Operative procedure of (AISE) in group A:}

For each expansion site, through $0.4 \mathrm{~cm}$ length incision made within the scar periphery or using the defect itself (post lesion excision) in the face, adequate blunt dissection by mosquito artery forceps for $3-4 \mathrm{~cm}$ were done. Then the silicone Foley catheter 14-16 Fr was probed in the dissected tract taking care to insert it with the balloon of the catheter become completely in the tract when inflated.

The balloon of the catheter was mildly filled and the dimensions just when we feel the edges of the balloon under the skin were firstly determined by the ruler and marked on the skin, this was considered the surface area of basic balloon dissection or basic skin stretch (BSS). Secondly, after emptying and removal of the balloon for the first time, the surface area of basic relaxing skin (BRS) was calculated from these dimensions on the skin.

Then the dissected area undergone 4 repeated cycles (C 1-4) of expansion with relaxing intervals for 3 minutes in-between cycles, in each cycle the balloon(s) of the catheter(s) synchronously were gradually inflated with $20 \mathrm{cc}$ saline then increased until the overlying skin was blanched and waiting for 5 minutes with expanded balloon and the dimensions the skin of maximal catheter dissection (MCD) were reported, then the balloon(s) were deflated and the generated skin after expansion (GSE) was measured. These dimensional measurements were determined and reported for the repeated 4cycles, then by comparing (BSS) to skin of C4 (MCD) to measure skin surface area gain by dissection \& stretch, and by comparing (BRS) to C4 (GSE) to measure skin surface area generated by expansion, and to calculate total skin surface area gain by dissection, stretch \& expansion. Then the generated skin was used in wound closure, combined with multi-planar injection of autologous 
fat harvested by the conventional techniques as in Figs. $(1,2)$.

\section{Operative procedure in group $B$ :}

They were managed by local flaps in (11 patients) such as conventional forehead flap in Fig. (3), or birhomboid flap as in Fig. (4) and full thickness skin graft was done in (3 patients).
The data of the two groups were collected regarding aetiology, operative time, common donor and recipient site complications: Early and late (two months) post-operative, tissue matching, patients' satisfaction, incidence of the need of secondary procedure. These determined data of both groups were compared and analyzed.
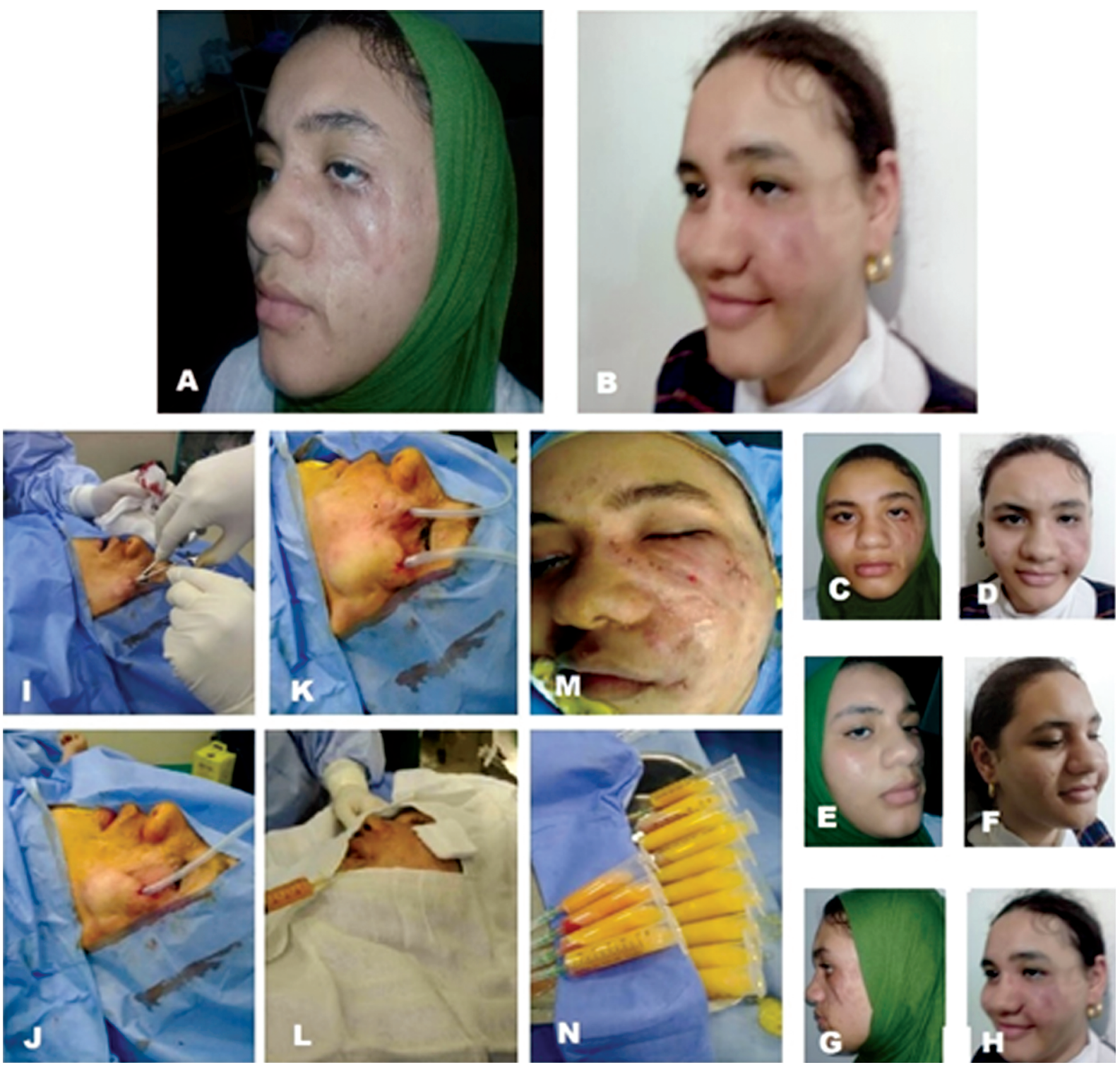

Fig. (1): Pre (A,C,E,G) and post-operative (B,D,F,H) views of 18 years old female with previously treated infantile haemangioma with marked fibrosis and lower lid ectropion, intraoperative views for the procedure of AISE for deficient skin and contracture $(\mathrm{I}, \mathrm{J}, \mathrm{K})$ associated with fat injection to correct contour irregularity. 

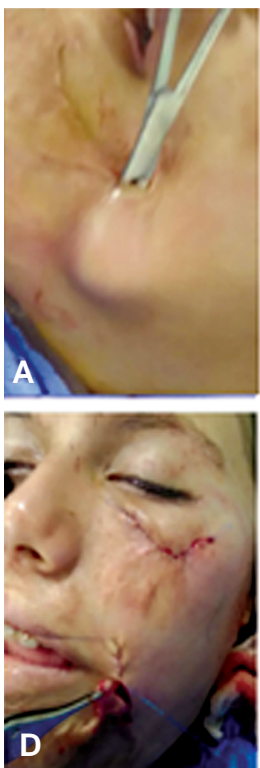
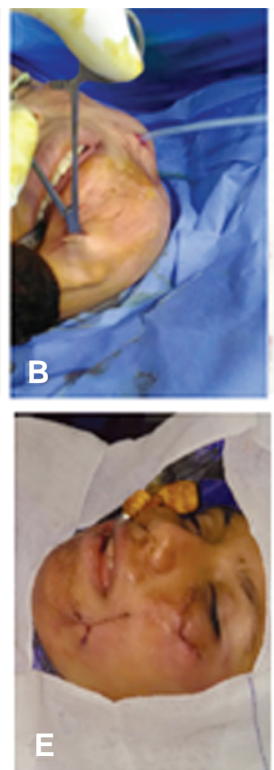
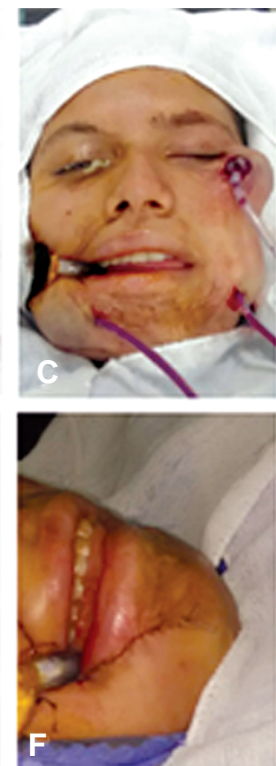
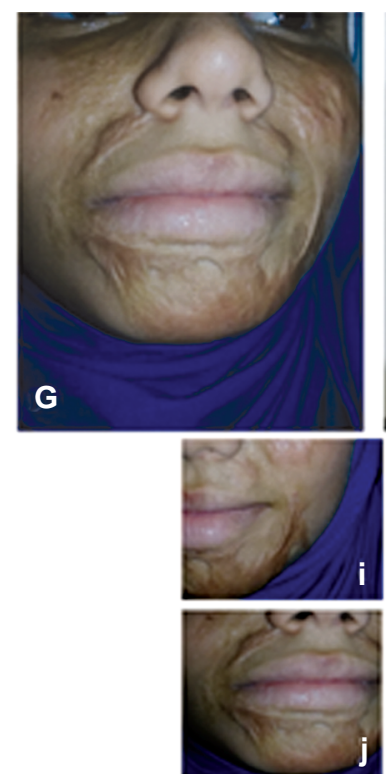

Fig. (2): Intra-operative views for the procedure of AISE (A,B,C,D,E,F), Preoperative (G,I,L) and post-operative (H,K,L) views of 15 years old female for correction perioral and cheek post burn contracture.
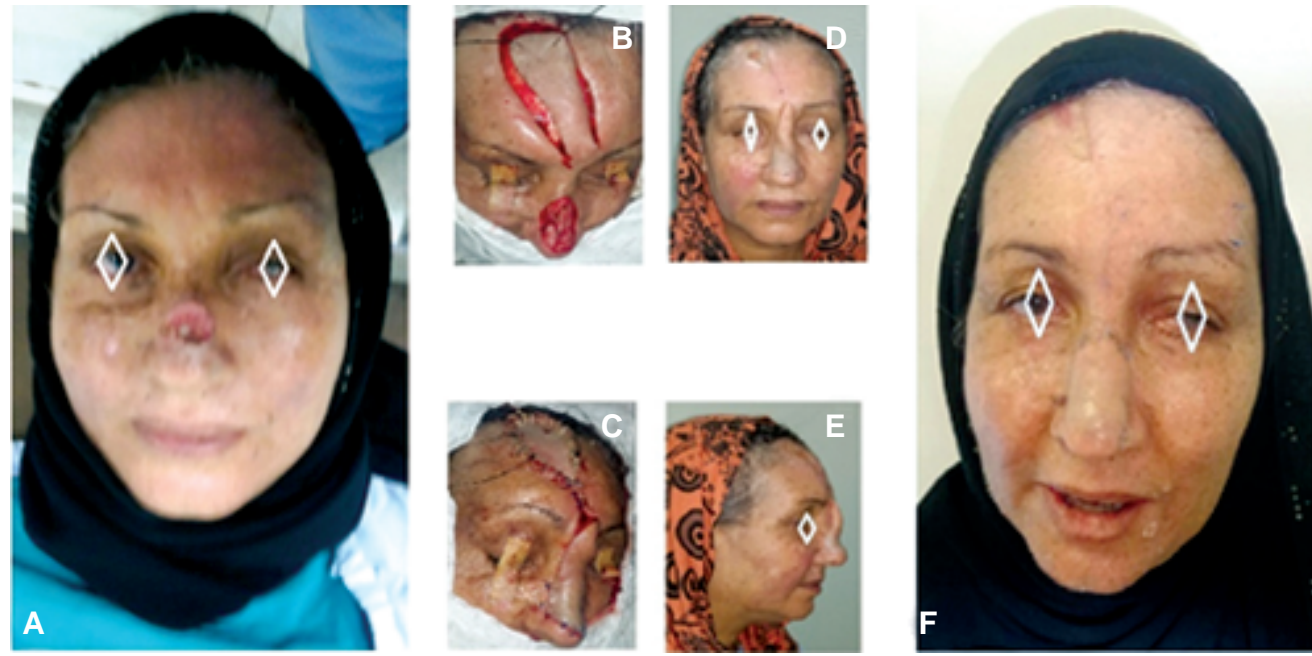

Fig. (3): A case of 65 years old female with nasal basal cell carcinoma. Post excision cutaneous defect $(3 \mathrm{~cm} \times 2,5)$ was reconstructed by pedicled forehead flap with grafting of the donor site. Preoperative (A), operative (B,C) and early post-operative views $(\mathrm{D}, \mathrm{E})$ before pedicle separation and late post-operative view $(\mathrm{F})$ after flap pedicle separation.
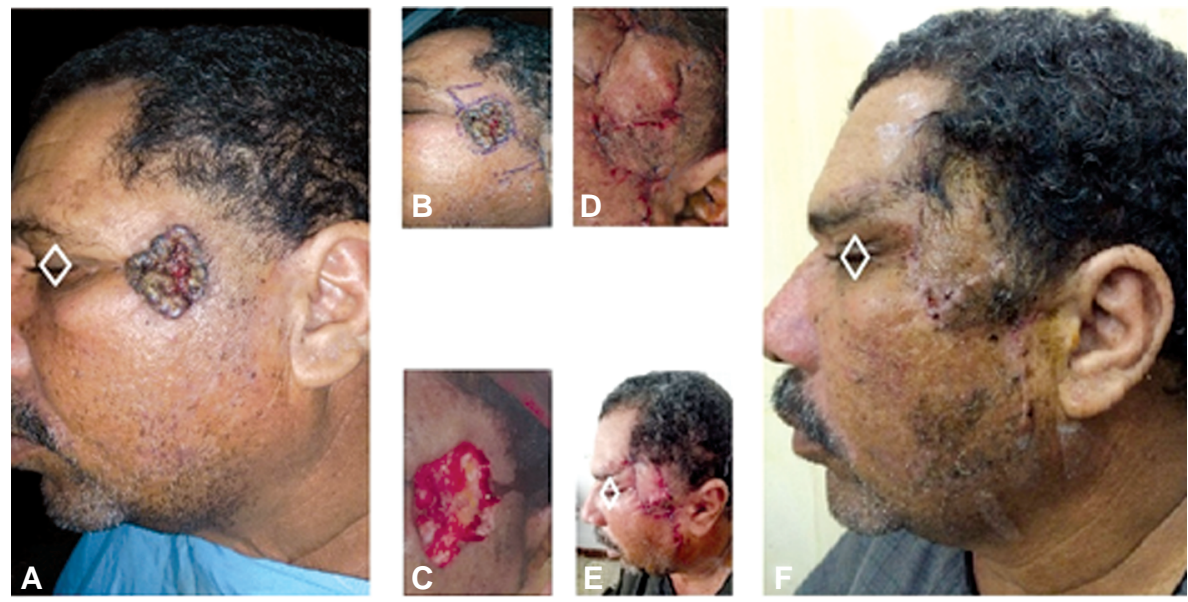

Fig. (4): A case of 61 years old man with basal cell carcinoma in the temple area, post excision cutaneous defect $(3 \times 3,5 \mathrm{~cm})$ was reconstructed by bi-rhomboid flap. Preoperative (A), operative flap design and excision, flap insitting (B,C,D) and early post-operative views (E) and late post-operative view (F) (note the distortion of hair bearing area). 


\section{RESULTS}

Regarding the (Expansion group), the 13 patients were treated by 21 Foley catheter expansion, by Comparing the mean operative time between the two groups, the expansion group (Group A) had more lengthy operation 140 minutes versus 75 minutes when treated with local flaps or grafts (Group B).

There were two cases of haematoma formation, 2 cases of closure under tension (one resulted in partial wound dehiscence) and 3 cases of patient dissatisfaction with the results. These complications were early in study and were a motive for us to refine the technique. However, the AISE showed success in closure of all defects or gaining skin in cases of contractures up to $3 \mathrm{~cm}$ length gap or 7 $\mathrm{cm}$ total surface area with each expansion site which is very precious skin in the face. Also, AISE was aesthetically reliable if associated with fat grafting in cases with contour defects.

Dimensional measurements were intraoperatively reported from all managed cases by AISE (Group A), the tissue response was obtained as follow Fig. (5): Percentage of skin surface area gained by dissection \& stretch ranged between 8.3$17.6 \%$ with the mean $14.2 \%$, percentage of skin surface area generated by expansion 10.1-19.3\% with the mean $16.5 \%$, and Percentage of total skin gain (total skin surface area gain by dissection, stretch \& expansion) ranged between 17.7-34.3\% with the mean $30.7 \%$.

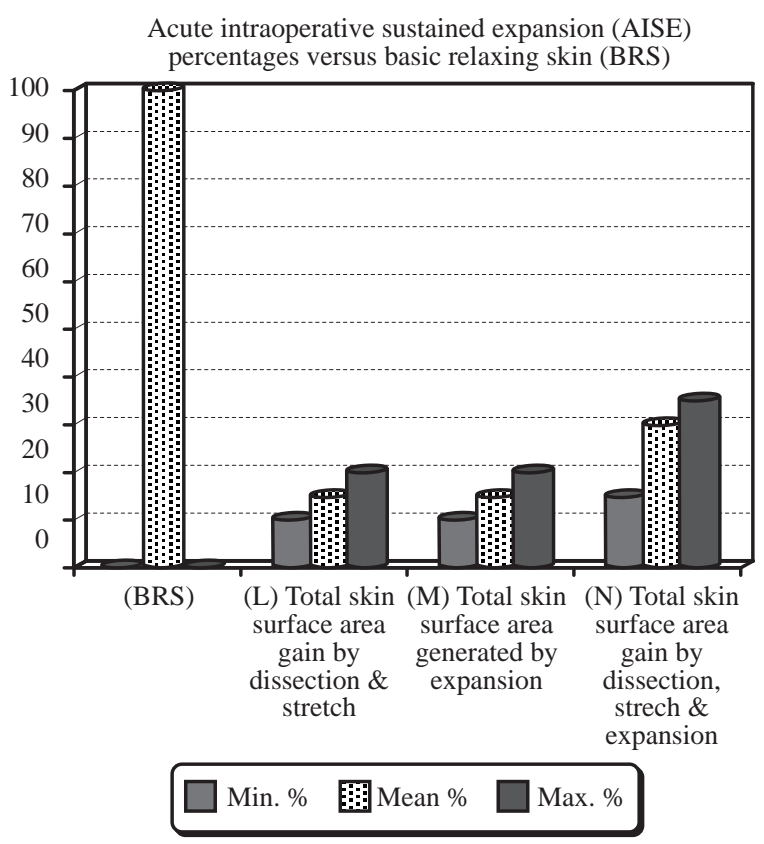

Fig. (5): Minimum, mean and maximum percentages of acute intraoperative sustained expansion (AISE) versus basic relaxing skin (BRS).
The most common complications in (Group B) were donor site unsatisfactory imaging in $80 \%$ of cases, wound dehiscence $22 \%$, need for second procedure $28 \%$, partial graft loss $20 \%$, unnatural distortion $65 \%$, poor color and texture matching to the surrounding area $25 \%$ in local flap cases and $100 \%$ in cases reconstructed by skin grafts, flap viability problems $12 \%$, additional facial scars $100 \%$ in cases of local flaps.

\section{DISCUSSION}

It is a great challenge to achieve a good or even accepted aesthetic outcome in patients with facial disfigurement.

Reconstruction either by flaps; local or distant, or by skin grafts was the common source of skin in facial resurfacing. However, the reconstruction by grafts usually results in undesirable results being non-sensate, with poor color, texture and characteristics matching [12], and it might be complicated by partial loss or contracture. On the other hand, flaps still have its own hazards regarding flap viability and the necessity to do another incision or more in the face with additional scars.

On the other hand, reconstruction by local tissue chronic expansion was described in literatures as having the privilege of generating a well color and characteristics matching skin. It is also having the advantages of obtaining a sensate skin and safer vascularity avoiding the complications of tissue transfer rendering it a good source of facial skin when needed $[\mathbf{1 2}, \mathbf{1 7}, \mathbf{1 8}]$.

In spite of three decades of worldwide research work in acute intraoperative immediate tissue expansion since its first description, its use in clinical work is still uncommon and relatively a rare procedure beside the chronic tissue expansion in literature all over the body. It may be due to that many surgeons tend to stick to the common practice $[15,19.20]$.

The use of chronic tissue expansion is commoner than the immediate one. However, several complications were reported with chronic expansion like potential infection risk which was described by some authors as non-significant problem [18]. Many other obstacles related to the implant itself like implant puncture, and port technical complications should be considered. Other drawbacks were including difficult to use in children, being two stages procedure having long period use with multiple follow-up clinic visits, producing soft tissue capsule in the bed and having a prolonged period of distortion annoying the patients. Compli- 
cations like erosion, hematoma and producing a significant pain were also reported [18] many of these disadvantages, could be circumvented by the use of AISE instead.

The available expanders are designed for single use and then must be discarded; so they are relatively expensive prosthesis. In the other hand, Foley catheters are disposable and inexpensive. Also, they are of immediate use with the advantages of absence of the complications of the prolonged implant use $[\mathbf{2 1 , 2 2 , 2 3 ]}$ or capsule formation being a completely intraoperative procedure [12].

Several authors had described the AISE technique by using Foley catheters in managing palatal defects ,after resection of alopecia or basal cell carcinoma or nevi masses in lid, temple or extremities $[\mathbf{1 2 , 1 5 , 1 6 ]}$. In this study, we also used Foley catheter in handling facial disfigurement, it was readily accessible, suitable, and practical device for intraoperative tissue expansion.

We agree with these notes with using Foley catheters and reported also less bleeding during some dissection procedures, less endangering the anatomical facial structures when done in the superficial subcutaneous plane and relatively rapid rising technical curve.

As regard mechanism of action, when applying chronic tissue expansion, the mechanism of tissue widening includes mitotic cellular changes, thinning of the dermis with a fibrosis around the expander capsule [10]. On the other hand, since Sasaki's initial report, the fundamentals of AISE are still underestimated, Hochman et al., claimed that the effect of minimizing wound closure tension by AISE was primarily owing to the undermining by implant and not the real tissue expansion itself [13].

Raposio and Bertozzi [14] had proved statistically significant biomechanical improvements by examining the ex-vivo biomechanical properties of acutely expanded skin flaps in fourteen fresh male cadavers.

They stated that as the cutaneous tissues possess both viscous and elastic properties, under low stress, it behaves like an elastic material, it demonstrates reversible immediate strain. However, under higher stress, it behaves like a viscous material, resulting in an increase in the skin length as a consequence of collagen fibers straightening and interstitial fluid displacement resulting in irreversible expansion; by a phenomenon called mechanical creep and stress relaxation [14].
Auletta et al., [15] reported 16-36\% tissue gain by intra-operative tissue expansion, beyond what was achieved by undermining alone in patients operated post Moh's surgery in the forehead, temple and scalp areas, he was able to close a $5 \mathrm{~cm}$ diameter facial skin defect. Others such as Baker and Swanson, and Johnson et al., achieved superior results and reported up to $31 \%$ tissue add with Intraoperative immediate tissue expansion $[\mathbf{1 6 , 1 9 , 2 0 ]}$.

Regarding this work, AISE showed success to gain up to $3 \mathrm{~cm}$ length skin or $7 \mathrm{~cm}$ total surface area with each expansion site by dissection, expansion, or both with means of $14.2 \%, 16.5 \%$ and $30.7 \%$ of the involved basic relaxing skin surface area respectively. although Foley catheter is available but the use of it is still considered by us as an immature instrumentation and in need for more modification to a specialized expander designed for intraoperative immediate tissue expansion with thick walled pressure bearing balloon, without tip and probe supported as we hope to share in it later on to overcome these technical difficulties.

Repeated inflation cycles in our experience showed more expandability than the first cycle, in agree with Johnson et al., [19], perhaps reorientation of fiber direction of the skin layers or fiber fatigue may be possibly the causes for further evaluation.

Also, this technique has shown another advantage by authors of providing less bleeding and easier dissection [19]. We agree with that concept, however early in the study, two expansion sites showed blood collection and discovered just postoperatively. Later we have considered that hemostasis should be carefully checked with superadded moderate compressive dressing for the first 24 hours postoperatively, especially if the drain is not preferred.

Conclusion: In selected cases, acute intraoperative sustained expansion can be a reliable reconstructive tool to facial disfigurements, generates a good matched skin in single procedure and more aesthetically reliable if associated with fat grafting injected in multiple subcutaneous planes in the same setting in cases with contour defects.

\section{REFERENCES}

1- Klein M.B.: Thermal, chemical and electrical injuries in Thorn C.H., Chung K.C., Gosein A.K., Gurtner G.C., Mehrara B.J., Rubin J.P. and Spear SL (eds). Grabb \& Smith's "Plastic Surgery", 6 ${ }^{\text {th }}$ edition, part 2, chapter, 15: 127-141, 2007.

2- Siemionov M., Sönmez E. and Papay A.: Principles of facial transplantation. In Weinzweig J, Plastic Surgery Secrets Plus, 2nd edition, chapter., 112: 724-728, 2010. 
3- Akhundzada I.S., Kerimov R.I., Aliyev A.A. and Huseynov T.A.: Supraclavicular flap for facial defects. AMAJ, 2: 45-49, 2016.

4- Spence R.J.: An algorithm for total and subtotal facial reconstruction using an expanded transposition flap: a 20-year experience. Plast. Reconstr. Surg., 121: 795-805, 2008.

5- Feldman J.J.: Facial burns. In: McCarthy J.G., editor. Plastic. Surgery. Philadelphia: PA: Saunders, p. 2153236, 1990.

6- Motamed S., Niazi F., Atarian S. and Motamed A.: Postburn head and neck reconstruction using tissue expanders. Burns, 34: 878-84, 2008.

7- Rivera R., LoGiudice J. and Gosain A.K.: Tissue expansion in pediatric patients. Clin. Plastic. Surg., 32: 35-44, 2005.

8- Heller L., Cole P. and kaufaman Y.: Cheek reconstruction: current concepts in managing facial soft tissue loss, Semin In Plastic. Surg. Nov., 22 (4): 294-305, 2008.

9- Joethy J. and Tan B.k.: A multi-staged approach to the reconstruction of a burnt Asian face, 44 (1): 142-46, 2011.

10- Gibstein L.A., Abramson D.L., Bartlett R.A., Orgill D.P., Upton J. and Mulliken J.B.: Tissue expansion in children: A reconstructive study of complications. Annals of Plastic Surgery, 100 (2): 297-304, 1997.

11- Sasaki G.H.: Intraoperative sustained limited expansion (ISLE) as an immediate reconstructive technique. Clin. Plast. Surg., 14: 563-573, 1987.

12- Abramo A.C., Viola J.C. and Angelo A.J.: Intraoperative rapid expansion in cleft palate repair. Plast. Reconstr. Surg., 91: 441-444, 1993.

13- Hochman M., Branham G. and Thomas J.R.: Relative effects of intraoperative tissue expansion on wound closing tension. Arch. Otolaryngol. Head Neck Surg., 118: 1185$1187,1992$.
14- Raposio E. and Bertozzi N.: Quantitative difference of acute intraoperative expansion in various body regions. European Review for Medical and Pharmacological Sciences, 21: 454-459, 2017.

15- Auletta M.J., Matarasso S.L., Glogau R.G. and Tromovitch T.A.: Comparison of skin hooks and Foley catheters for immediate tissue expansion. J. Dermatol. Surg. Oncol., 19: 1084-1088, 1993.

16- Cil Y., Emre A. and Ozturk S.: Dissection of the expander pocket for burn scar alopesia treatment with the aid of urologic instrument and a foley catheter. J. Burn. Care. Res., 29: 681-681, 2008.

17- Foster J.A., Scheiner A.J., Wulc A.E., Wallace I.B. and Greenbaum S.S.: Intraoperative tissue expansion in eyelid reconstruction. Ophthalmology, 105: 170-175, 1998.

18- Friedman R.M., Ingram A.E.Jr., Rohrich R.J., Byrd H.S., Hodges P.L., Burns A.J. and Hobar P.C.: Risk factors for complications in pediatric tissue expansion. Plast. Reconstr. Surg., 98: 1242-1246, 1996.

19- Johnson T.M., Brown M.D., Sullivan M.J. and Swanson N.A.: Immediate intraoperative tissue expansion. J. Am. Acad. Dermatol., 22: 283-287, 1990.

20- Baker S.R. and Swanson N.A.: Rapid intraoperative tissue expansion in reconstruction of the head and neck. Arch. Otolaryngol. Head Neck Surg., 116: 1431-1434, 1990.

21- Kolbusz R.V. and Bielinski K.B.: Basal cell carcinoma: Excision with immediate intraoperative tissue expansion. Cutis., 46: 419-420, 1990.

22- Greenbaum S.S.: Intraoperative tissue expansion with the Foley catheter. J. Dermatol. Surg. Oncol., 19: 1079-1083, 1993.

23- Greenbaum S.S. and Greenbaum C.H.: Intraoperative tissue expansion using a Foley catheter following excision of a basal cell carcinoma. J. Dermatol. Surg. Oncol., 16: 45-48, 1990. 